\title{
A MESOSCOPIC THEORY OF DAMAGE AND FRACTURE IN HETEROGENEOUS MATERIALS
}

\author{
A. Carpinteri, B. Chiaia and P. Cornetti \\ Department of Structural Engineering and Geotechnics \\ Politecnico di Torino, 10128 Torino, Italy
}

\begin{abstract}
Deformation patterns in solids are often characterized by self-similarity at the meso-level. In this paper, the framework for the mechanics of heterogeneous solids, deformable over fractal subsets, is briefly outlined. Mechanical quantities with non-integer physical dimensions are considered, i.e., the fractal stress [ $\left.\sigma^{*}\right]$ and the fractal strain $\left[\varepsilon^{*}\right.$. By means of the local fractional calculus, the static and kinematic equations are obtained. The extension of the Gauss-Green Theorem to fractional operators permits to demonstrate the Principle of Virtual Work for fractal media. From the definition of the fractal elastic potential $\phi^{*}$, the fractal linear elastic relation is derived. Beyond the elastic limit, peculiar mechanisms of energy dissipation come into play, providing the softening behaviour characterized by the fractal fracture energy $\mathrm{G}_{F}{ }^{*}$. The entire process of deformation in heterogeneous bodies can thus be described by the fractal theory. In terms of the fractal quantities it is possible to define a scale-independent cohesive law which represents a true material property. It is also possible to calculate the size-dependence of the nominal quantities and, in particular, the scaling of the critical displacement $w_{c}$, which explains the increasing tail of the cohesive law with specimen size, and that of the critical strain $\varepsilon_{c}$, which explains the brittleness increase with specimen size.
\end{abstract}

\section{KEYWORDS}

Mesomechanics, fractals, fractional calculus, cohesive crack model, size-effects.

\section{INTRODUCTION: FRACTAL STRESS AND FRACTAL STRAIN}

The singular stress flux through fractal media can be modelled by means of a lacunar fractal set $A^{*}$ of dimension $\Delta_{\sigma}$, with $\Delta_{\sigma} \leq 2$. An original definition of the fractal stress $\sigma^{*}$ acting upon lacunar domains was put forward by Carpinteri [1] by applying the renormalization group procedure to the nominal stress tensor $[\sigma]$. The fractal stress $\sigma^{*}$, whose dimensions are $[\mathrm{F}][\mathrm{L}]^{-(2-d \sigma)}$, is a scale-invariant quantity. For simplicity, a uniaxial tensile field is considered in Figure 1. Note that, for the definition of $\sigma^{*}$, exactly as in the case of the classical Cauchy stress, the limit:

$$
\lim _{\Delta A^{*} \rightarrow 0}\left(\Delta P / \Delta A^{*}\right)
$$

is supposed to exist and, eventually, to attain finite values at any singular point of the support $A^{*}$. This is mathematically possible for lacunar sets like that in Figure 1 (and also for rarefied point sets like Cantor sets) which, although not compact, are dense in the surrounding of any singular point. 

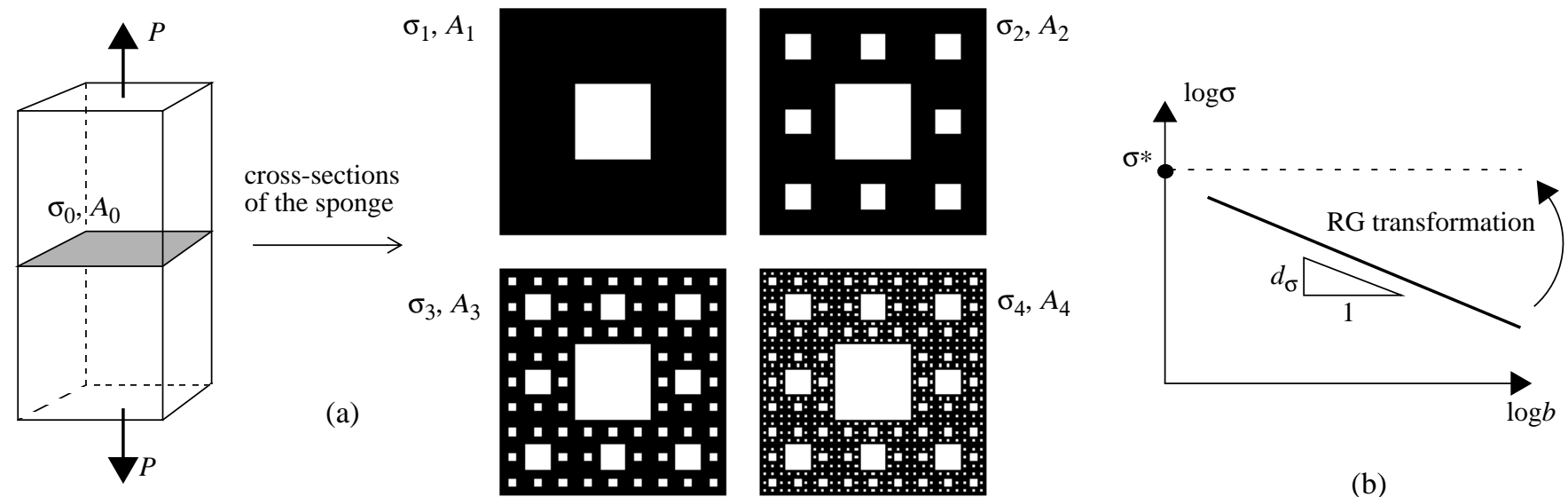

(b)

$$
P=\sigma_{0} A_{0}=\sigma_{1} A_{1}=\sigma_{2} A_{2}=\ldots=\sigma_{n} A_{n}=\ldots=\sigma^{*} b^{2-d_{\sigma}}
$$

Figure 1. Renormalization of the stress over a Sierpinski carpet (a) and scaling of the nominal stress (b).

The kinematical counterpart of the fractal stress is the fractal strain $\varepsilon^{*}$. The starting assumption is that displacement discontinuities can be localized on an infinite number of cross-sections, spreading throughout the body [2]. Experimental investigations confirm the fractal character of deformation, for instance in metals (slip lines with cantorian structure [3]), and in highly stressed rock masses (plastic shear bands).

Considering the simplest uniaxial model, a slender bar subjected to tension, it can be argued that the horizontal projection of the cross-sections where deformation localizes is a lacunar fractal set, with dimension between zero and one. If the Cantor set $\left(\Delta_{\varepsilon} \cong 0.631\right)$ is assumed as an archetype of the damage distribution, we may speak of the fractal Cantor bar (Figure 2a). The dilation strain tends to concentrate into singular stretched regions, while the rest of the body is practically undeformed. The displacement function can be represented by a devil's staircase graph, that is, by a singular fractal function which is constant everywhere except at the points corresponding to a lacunar fractal set of zero Lebesgue measure (Figure 2b).

(a)

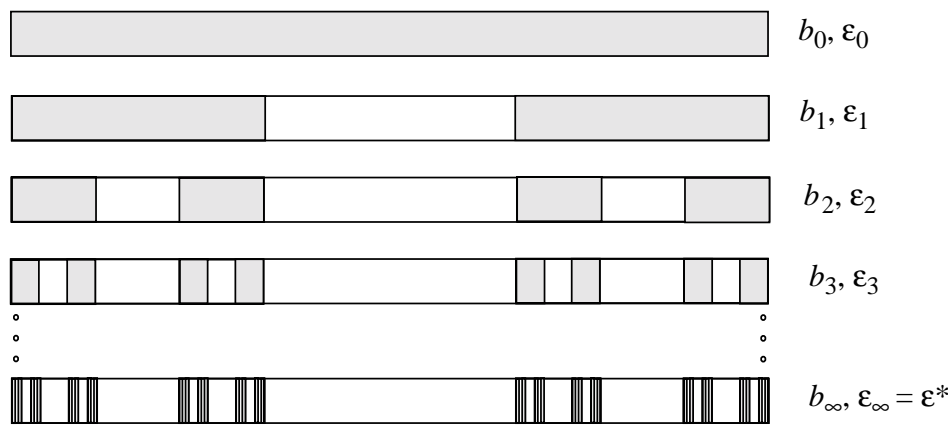

$w=\varepsilon_{0} b_{0}=\varepsilon_{1} b_{1}=\varepsilon_{2} b_{2}=\ldots=\varepsilon_{n} b_{n}=\ldots=\varepsilon^{*} b_{0}^{1-d} \varepsilon$ (b)

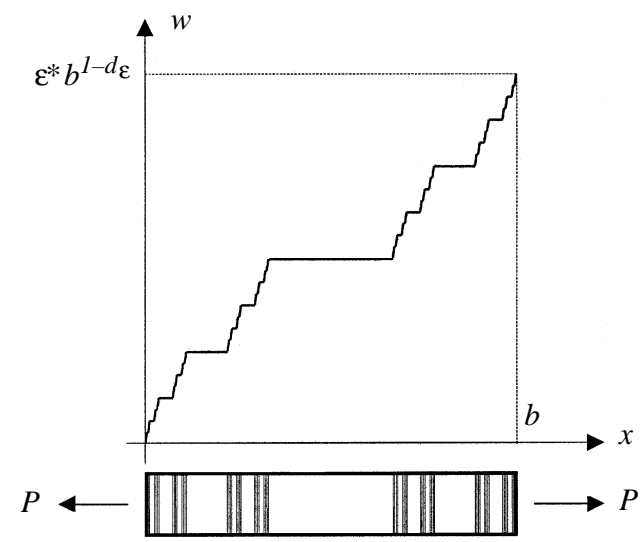

Figure 2. Renormalization of the strain over a Cantor bar (a) and singular displacement function (b).

Let $\Delta_{\varepsilon}=1-d_{\varepsilon}$ be the fractal dimension of the lacunar projection of the deformed sections. Since $\Delta_{\varepsilon} \leq 1$, the fractional decrement $d_{\varepsilon}$ is always a number between 0.0 (corresponding to strain smeared along the bar) and 1.0 (corresponding to the maximum localization of strain, i.e., to localized fracture surfaces). By applying the renormalization group procedure (see Figure 2a), the micro-scale description of displacement requires the product of the fractal strain $\varepsilon^{*}$ times the fractal measure $b_{0}{ }^{\left(1-d_{\varepsilon}\right)}$ of the support. The fractal strain $\varepsilon^{*}$ is the scale-independent parameter describing the kinematics of the fractal bar. Its physical dimensionality [L] ${ }^{d}{ }_{\varepsilon}$ is intermediate between that of a pure strain $[\mathrm{L}]^{0}$ and that of a displacement $[\mathrm{L}]$, and synthesizes the conceptual transition between classical continuum mechanics $\left(d_{\varepsilon}=0\right)$ and fracture mechanics $\left(d_{\varepsilon}=1\right)$. Correspondingly, the kinematical controlling parameter changes, from the nominal strain $\varepsilon$, to the crack opening displacement $w$. By varying the value of $d_{\varepsilon}$ (e.g. for different loading levels), the evolution of strain localization can be cap- 
tured. The two limit situations are shown in Figure 3, the devil's staircase being an intermediate situation with $d_{\varepsilon} \cong 0.369$. While the first case represents the classical homogeneous elastic strain field, the second diagram shows a single displacement discontinuity, e.g., the formation of a sharp fracture.

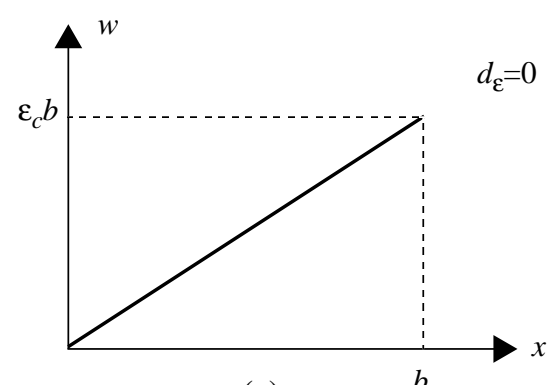

(a)

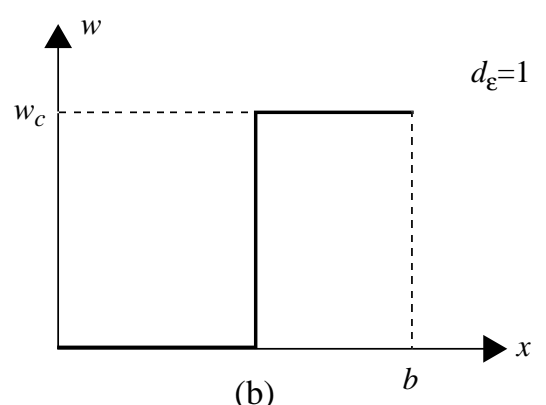

(b)

Figure 3. Homogeneous strain (a) and extremely localized deformation (b) over the bar (critical point).

During a generic loading process, the mechanical work $W^{*}$ can be stored in the body as elastic strain energy (conservative process) or dissipated on the infinite lacunar cross-sections where strain is localized (dissipative process). In any case, the fractal domain $\Omega^{*}$, with dimension $3-d_{\omega}$, where the mechanical work is produced, must be equal to the cartesian product of the lacunar cross-section with dimension $2-d_{\sigma}$, times its cantorian projection with dimension $1-d_{\varepsilon}$. Since the dimension of the product of two fractal sets is equal to the sum of their dimensions, one obtains: $\left(3-d_{\omega}\right)=\left(2-d_{\sigma}\right)+\left(1-d_{\varepsilon}\right)$, which yields the fundamental relation among the exponents as:

$$
d_{\omega}=d_{\sigma}+d_{\varepsilon}
$$

\section{STATIC AND KINEMATIC EQUATIONS FOR FRACTAL MEDIA}

Classical fractional calculus is based on nonlocal operators. Recently, Kolwankar and Gangal [4] have introduced a new operator called local fractional integral. Let $\left[x_{i}, x_{i+1}\right], i=0, \ldots, N-1, x_{0}=a, x_{N}=b$, be a partition of the interval $[a, b]$, and $x_{i}^{*}$ some suitable point of the interval $\left[x_{i}, x_{i+1}\right]$. Consider then a function $f(x)$ defined on a lacunar fractal set belonging to $[a, b]$. The fractal integral of order $\alpha$ of the function $f(x)$ over the interval $[a, b]$ is defined as:

$$
I^{\alpha}[f(x)]_{a}^{b}=\lim _{N \rightarrow \infty} \sum_{i=0}^{N-1} f\left(x_{i}^{*}\right) \frac{\mathrm{d}^{-\alpha} 1_{d x_{i}}(x)}{\left[\mathrm{d}\left(x_{i+1}-x_{i}\right)\right]^{-\alpha}},
$$

where $1_{d x_{i}}(x)$ is the unit function defined upon $\left[x_{i}, x_{i+1}\right]$. The fractal integral is a mathematical tool suitable for the computation of fractal measures. In fact, it yields finite values of the measure if and only if the order of integration is equal to the dimension of the fractal support of function $f(x)$. Otherwise, its value is zero or infinite, thus showing a behaviour analogous to the Hausdorff measure of a fractal set. Kolwankar and Gangal [4] introduced also the local fractional derivative (LFD) of order $\alpha$, whose definition is $(0<\alpha<1)$ :

$$
D^{\alpha} f(y)=\lim _{x \rightarrow y} \frac{\mathrm{d}^{\alpha}[f(x)-f(y)]}{\mathrm{d}(x-y)^{\alpha}} .
$$

Differently from the classical fractional derivative, the LFD is a function only of the $f(x)$ values in the neighborhood of the point $y$ where it is calculated. The classical fractional derivative of a fractal function exists as long as its order is less than the Hölder exponent characterizing the singularity. Instead, in the singular points, the LFD (Eqn. (4)) is generally zero or infinite. It assumes a finite value only if the order $\alpha$ of derivation is exactly equal to the Hölder exponent of the graph. For instance, in the case of the well-known devil's staircase graph (Figure $2 b$ ) the LFD of order $\alpha=\log 2 / \log 3$ (i.e. equal to the dimension of the underlying middlethird Cantor set) is zero everywhere except in the singularity points where it is finite. 
By means of the LFD, the fractal differential equations of kinematics and statics can be obtained [5]. The displacement field maintains the dimension of length. The noninteger dimensions of the fractal strain are: [L] $]^{d \varepsilon}$. Therefore, it can be obtained by fractional differentiation of the displacement vector $\{\eta\}$, according to the definition of LFD outlined above. The fractional differential operator $\left[\partial^{\alpha}\right]$ can be introduced, where the order of differentiation is $\alpha=1-d_{\varepsilon}$. Thereby, the kinematic equations for the fractal medium can be written, in the vector notation, as:

$$
\left\{\varepsilon^{*}\right\}=\left[\partial^{\alpha}\right]\{\eta\}
$$

Classical strain is obtained when $\alpha=1\left(d_{\varepsilon}=0\right)$. Instead, when $\alpha=0$, strain is no longer homogeneously diffused and reduces to localized displacement discontinuities. The intermediate situations are described by generic values of $\alpha$.

The static equations link the fractal stress vector $\left\{\sigma^{*}\right\}$ to the vector of body forces $\left\{\mathcal{F}^{*}\right\}$, which assumes noninteger dimensions according to the fractal dimension of the deformable subset $\Omega^{*},[\mathrm{~F}][\mathrm{L}]^{-\left(3-d_{\omega}\right)}$. On the other hand, the dimensions of the fractal stress are $[\mathrm{F}][\mathrm{L}]^{-(2-d \sigma)}$. Therefore, the equilibrium equations can be written, in the vector notation, as:

$$
\left[\partial^{\alpha}\right]^{T}\left\{\sigma^{*}\right\}=-\left\{\mathcal{F}^{*}\right\}
$$

where the static fractional differential operator $\left[\partial^{\alpha}\right]^{T}$ is the transposed of the kinematic fractional differential operator $\left[\partial^{\alpha}\right]$. It is worth to observe that the fractional order of differentiation of the static operator in the fractal medium is $\alpha=1-d_{\varepsilon}$, the same as that of the kinematic operator (Eqn. (5)). This remarkable result is due to the fundamental relation among the exponents (Eqn. (2)), and represents the Duality Principle for Fractal Media. Finally, equivalence at the boundary of the body requires that the stress vector coincides with the applied fractal boundary forces $\left\{p^{*}\right\}$ (with physical dimensions $[\mathrm{F}][\mathrm{L}]^{-(2-d \sigma)}$ ):

$$
[\mathcal{N}]^{T}\left\{\sigma^{*}\right\}=\left\{p^{*}\right\} .
$$

In the case of fractal bodies, $[\mathcal{N}]^{T}$ can be defined, at any dense point of the boundary, as the cosine matrix of the outward normal to the boundary of the initiator of the fractal body.

\section{PRINCIPLE OF VIRTUAL WORK AND LINEAR ELASTIC LAW FOR FRACTAL MEDIA}

Consider two arbitrary functions $f(x, y, z)$ and $g(x, y, z)$, defined in a fractal domain $\Omega^{*}$, with the same critical order $\alpha$. The general formula of local fractional integration by parts has been obtained by the authors [5] as:

$$
\left\{I^{(\beta-\alpha)}[g f]\right\}_{\Gamma^{*}}=\left\{I^{\beta}\left[g D^{\alpha} f\right]\right\}_{\Omega^{*}}+\left\{I^{\beta}\left[f D^{\alpha} g\right]\right\}_{\Omega^{*}},
$$

where $\Gamma^{*}$ is the boundary of the domain $\Omega^{*}$. This result extends the Gauss-Green Theorem to 3D fractal domains. Based on Eqn. (8), the Principle of Virtual Work for fractal media was demonstrated [5]. It reads:

$$
\int_{\Omega^{*}\left(3-d_{\omega}\right)}\left\{\mathcal{F}_{A}^{*}\right\}^{T}\left\{\eta_{B}\right\} \mathrm{d} \Omega^{*}+\int_{\Gamma^{*}\left(2-d_{\sigma}\right)}\left\{p^{*}\right\}^{T}\left\{\eta_{B}\right\} \mathrm{d} \Gamma^{*}=\int_{\Omega^{*}\left(3-d_{\omega}\right)}\left\{\sigma^{*}\right\}^{T}\left\{\varepsilon^{*}{ }_{B}\right\} \mathrm{d} \Omega^{*}
$$

Both sides of Eqn. (9) possess the dimensions of work ([F][L]), since the operators are fractional integrals defined upon fractal domains. The external work may be done by fractal body forces $\left\{\mathcal{F}^{*}\right\}$ and/or by fractal tractions $\left\{p^{*}\right\}$ acting upon the boundary $\Gamma^{*}$ of the body. The internal work of deformation is defined as: $\mathrm{d} W^{*}=\left\{\sigma^{*}\right\}^{T}\left\{\mathrm{~d} \varepsilon^{*}\right\}$, with dimensions $[\mathrm{F}][\mathrm{L}]^{-\left(2-d_{\omega}\right)}$. If the (initial) loading process is conservative (no dissipation occurs in the material), and stress is a univocal function of strain, a fractal elastic potential $\phi^{*}$ (function of the fractal strain $\left.\left\{\varepsilon^{*}\right\}\right)$ can be considered. The components of the fractal stress vector $\left\{\sigma^{*}\right\}$ can therefore be obtained by derivation: 


$$
\sigma_{i}^{*}=\frac{\partial \phi^{*}}{\partial \varepsilon_{i}^{*}}
$$

Note that these are canonical first-order partial derivatives in the space of the fractal strains $\left\{\varepsilon^{*}\right\}$. Performing the Taylor expansion around the undeformed state, and neglecting higher order derivatives, the following bilinear form can be easily obtained:

$$
\phi^{*}=\frac{1}{2}\left\{\varepsilon^{*}\right\}^{T}\left[\boldsymbol{H}^{*}\right]\left\{\varepsilon^{*}\right\},
$$

where $\left[\boldsymbol{H}^{*}\right]$ is the Hessian matrix of the fractal elastic potential. Dimensional arguments show that the anomalous dimensions of $\left[\boldsymbol{H}^{*}\right]$ are: $[F][L]^{-\left(2+d_{\varepsilon}-d_{\sigma}\right)}$. Thus, $\left[\boldsymbol{H}^{*}\right]$ depends on both the dimensions of stress and strain and, depending on the difference $\left(d_{\sigma}-d_{\varepsilon}\right)$, can be subjected to positive or negative size-effects. Each term in $\left[\boldsymbol{H}^{*}\right]$ is obtained as the second-order partial derivative of the elastic potential by the corresponding fractal strain:

$$
\boldsymbol{H}^{*}{ }_{i j}=\frac{\partial^{2} \phi^{*}}{\partial \varepsilon_{i}^{*} \partial \varepsilon_{j}^{*}} .
$$

From Eqns. (10) and (11), the linear elastic constitutive law for fractal media is provided as:

$$
\left\{\sigma^{*}\right\}=\left[\boldsymbol{H}^{*}\right]\left\{\varepsilon^{*}\right\}
$$

\section{SCALE INDEPENDENT COHESIVE CRACK LAW}

After the initial elastic stage, when $d_{\varepsilon}$ is close to 0 , a nonlinear stage occurs, where damage and microcracking begin to spread and $d_{\varepsilon}$ grows. In concrete-like materials, strain localizes quite soon in a band, and the softening stage comes into play. The cohesive law describes the decrement of the stress as a function of crack opening displacement $w$. The original model is based on the assumption that both the critical crack opening displacement $w_{c}$ and the ultimate strength $\sigma_{u}$ are independent of the structural size. Unfortunately, experiments show that this is not the case. Moreover, it is well-known that the area below the cohesive curve, i.e., the fracture energy $\mathrm{G}_{F}$, is subjected to relevant positive size-effects [6].
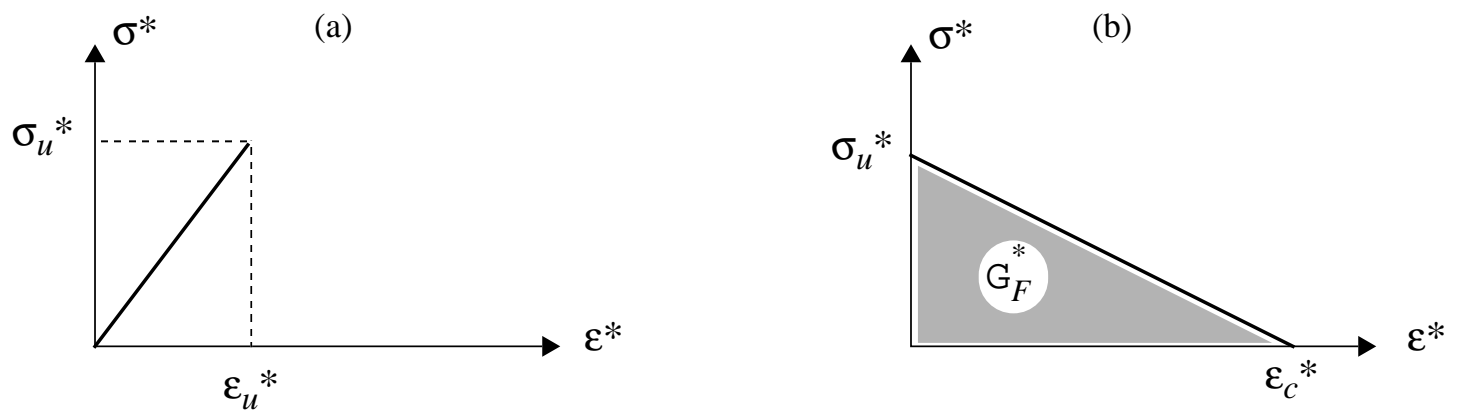

Figure 4. Fractal elastic law (a), and fractal cohesive law (b).

To overcome this limitation, the model associates to the fractal linear elastic law valid for the undamaged material (Figure 4a), a softening relationship between fractal stress and fractal strain, assuming that $\sigma_{u}{ }^{*}$ and $\varepsilon_{C}{ }^{*}$ are the true scale-independent limit parameters. It is interesting to note that the fractal fracture energy $\mathrm{G}_{F}^{*}$, defined in [1,6], can be obtained, by a fractional integral, as the area below the fractal softening diagram (Figure 4b):

$$
\mathrm{G}_{F}^{*}=\int_{0}^{\varepsilon^{*}} \sigma^{*} \mathrm{~d} \varepsilon^{*}
$$


During the softening regime, i.e. when most of the dissipation occurs, $\sigma^{*}$ decreases from the maximum value $\sigma_{u}{ }^{*}$ to 0 , while $\varepsilon^{*}$ grows up to $\varepsilon_{c}^{*}$. In the meantime, the non-damaged parts of the bar undergo elastic unloading. We call the $\sigma^{*}-\varepsilon^{*}$ diagram the fractal cohesive law, which is shown in Figure $4 \mathrm{~b}$. Contrarily to the classical cohesive law, which is sensitive to the structural size, this curve is scale-independent. Experimental tests by van Mier \& van Vliet [7] have shown that, with increasing the specimen size, the peak of the curve decreases whereas the tail rises, i.e., tensile strength decreases while critical displacement increases. More in detail, $w_{c}$ varies more rapidly than $\sigma_{u}$ does. Therefore, an increase of the area beneath the cohesive law, i.e. of the fracture energy, is observed. Thus, the experimental trends of $\sigma_{u}, G_{F}$ and $w_{c}$ confirm the assumptions of the fractal model.

(a)

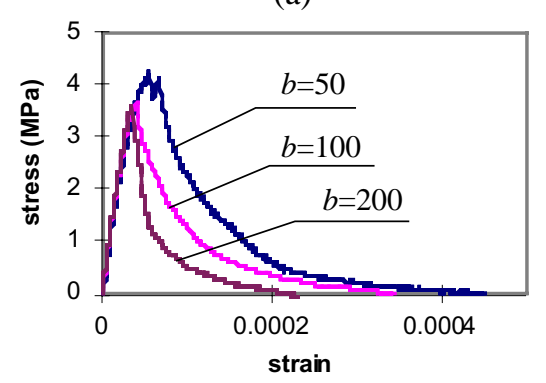

(b)

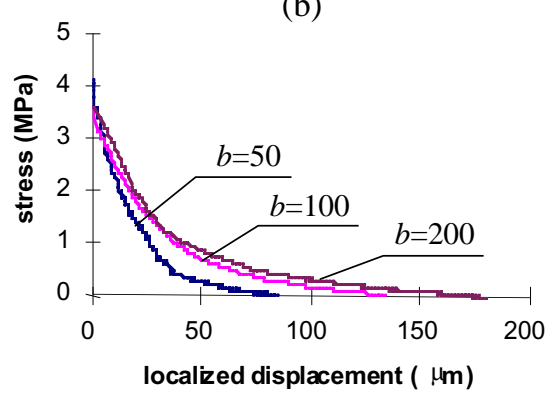

(c)

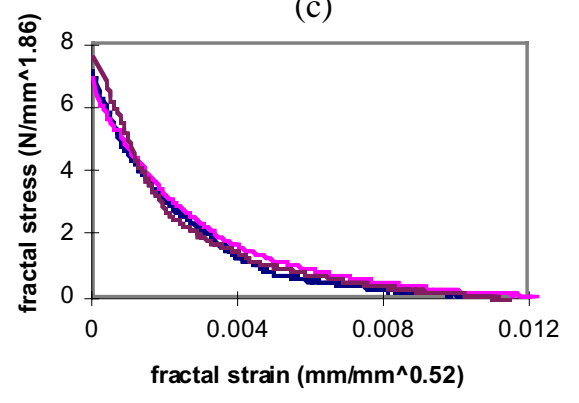

Figure 5. Size-effect tests [7]: stress-strain diagrams (a), cohesive curves (b) and fractal cohesive law (c).

The fractal model has been eventually compared with the uniaxial tensile tests described in [8]. The attention was focused on the size-effect on the ultimate tensile stress and on the fracture energy and their values interpreted by means of fractal assumptions. The exponents of the scaling laws were deduced by fitting the experimental results. In particular, they found the values $d_{\sigma}=0.14$ and $d_{\mathrm{G}}=0.38$. The nominal $\sigma-\varepsilon$ and $\sigma-w$ diagrams are reported in Figure 5a and 5b. Here, $w$ is the displacement localized in the damage band, obtained by subtracting from the total one the displacement due to elastic and inelastic pre-peak deformation. In addition, the value $d_{\varepsilon}=0.48$ is provided by Eqn. (2), so that the fractal cohesive laws can be represented as in Figure 5c. As expected, all the curves related to the single sizes tend to merge in a unique, scale-independent cohesive law.

\section{ACKNOWLEDGEMENTS}

Supports by the Italian Ministry of University and Scientific Research and by the EC-TMR Contract $\mathrm{N}^{\circ}$ ERBFMRXCT 960062, are gratefully acknowledged.

\section{REFERENCES}

1. A. Carpinteri (1994) Mechanics of Materials 18, 89.

2. P. Cornetti (1999) Fractals and Fractional Calculus in the Mechanics of Damaged Solids, Ph.D. Thesis (in Italian). Politecnico di Torino, Torino.

3. T. Kleiser and M. Bocek (1986) Zeitschrift für Metallkunde 77, 582.

4. K.M. Kolwankar and A.D. Gangal (1996) Chaos 6, 505.

5. A. Carpinteri, B. Chiaia and P. Cornetti (2001) Computer Methods in Applied Mechanics and Engineering, in print.

6. A. Carpinteri and B. Chiaia (1995) Material and Structures 28, 435.

7. J.G.M. van Mier and M.R.A. van Vliet (1999) International Journal of Fracture 94, 195.

8. A. Carpinteri and G. Ferro (1994) Materials and Structures 27, 563. 